\title{
Role of Oxidative Stress and Severity of Diabetic Retinopathy in Type 1 and Type 2 Diabetes
}

\author{
Kholoud Bokhary $^{\mathrm{a}}$ Feda Aljaser $^{\mathrm{b}}$ Manal Abudawood $^{\mathrm{b}}$ Hajera Tabassum $^{\mathrm{d}}$ \\ Afnan Bakhsh $^{d}$ Shatha Alhammad ${ }^{\mathrm{a}}$ Rawan Aleyadhi ${ }^{\mathrm{a}}$ Faisal Almajed $^{\mathrm{c}}$ \\ Roua Alsubki ${ }^{b}$ \\ aDepartment of Optometry and Vision Science, College of Applied Medical Sciences, King Saud University, Riyadh, \\ Kingdom of Saudi Arabia; ' Department of Clinical Laboratory Sciences, Chair of Medical and Molecular Genetics, \\ College of Applied Medical Sciences, King Saud University, Riyadh, Kingdom of Saudi Arabia; 'Department of \\ Clinical Laboratory Sciences, College of Applied Medical Sciences, King Saud Bin Abdulaziz University for Health \\ Sciences, Riyadh, Kingdom of Saudi Arabia; 'Department of Clinical Laboratory Sciences, College of Applied Medical \\ Sciences, King Saud University, Riyadh, Kingdom of Saudi Arabia
}

\section{Keywords}

Oxidative stress - Diabetic retinopathy $\cdot$ Antioxidant

\begin{abstract}
Background: Diabetic retinopathy (DR) is a sight-threatening complication of diabetes mellitus (DM). Oxidative stress generated on account of hyperglycemic state may lead to retinal abnormalities including DR. Objectives: The aim of the study was to evaluate the status of antioxidant enzymes; superoxide dismutase (SOD), and catalase (CAT), in different stages of DR severity in subjects with type 1 DM (T1DM) and type 2 DM (T2DM). Methods: The cross-sectional study enrolled 148 subjects with T1DM $(n=17), \operatorname{T2DM}(n=96)$, and nondiabetic controls $(n=35)$. Subjects with DM were divided into 2 subgroups based on DR severity (mild-to-severe nonproliferative DR [NPDR] and proliferative DR [PDR]), and serum glycated hemoglobin $(\mathrm{HbA} 1 \mathrm{c})$, lipid profile, SOD, and CAT were estimated. Results: Both SOD and CAT levels were lower in diabetic subjects than nondiabetic controls. A significant positive correlation was found between $\mathrm{HbA} 1 \mathrm{C}$ lev-
\end{abstract}

karger@karger.com www.karger.com/ore

Karger!"
(C) 2021 The Author(s)

Published by S. Karger AG, Basel

This is an Open Access article licensed under the Creative Commons Attribution-NonCommercial-4.0 International License (CC BY-NC) (http://www.karger.com/Services/OpenAccessLicense), applicable to the online version of the article only. Usage and distribution for commercial purposes requires written permission. el and severity of DR ( $p<0.0001)$. Levels of SOD and CAT varied significantly with DR severity in both diabetic groups at $p<0.05$. Furthermore, levels of SOD and CAT were found to decrease significantly $(p<0.001)$ in DR $(+)$ compared to DR (-) patients. Also, increased levels of $\mathrm{HbA} 1 \mathrm{c}$ were significantly associated $(p<0.001)$ with decreased SOD in both subgroups (NPDR and PDR). DR severity was significantly associated with SOD and CAT in the NPDR and PDR subgroups $(p<0.05)$. Conclusion: Oxidative stress and decreased antioxidant defenses are associated with DR progression to its PDR stage.

(c) 2021 The Author(s). Published by S. Karger AG, Basel

\section{Introduction}

Vision loss or impairment is one of the consequences of diabetic retinopathy (DR) resulting from untreated diabetes mellitus (DM). DM is characterized by hyperglycemia which is a condition of elevated blood glucose levels caused by insufficient insulin secretion from pancre- 
atic beta cells (type $1 \mathrm{DM}$ [T1DM]) or unresponsiveness of various receptor cells to insulin (type $2 \mathrm{DM}$ [T2DM]). $\mathrm{DR}$ can occur in both diabetes types and is strictly related to disease duration. Glycated hemoglobin (HbAlc) is a major determinant of hyperglycemia and is formed because of glucose overload in the blood [1]. Hyperglycemic conditions have harmful effects on the microvasculature of different body parts, especially the eyes. Microvascular abnormalities in the retina lead to many abnormalities, including neovascularization, macular edema, and retinal detachment [2]. These abnormalities are markers of retinal damage caused by hyperglycemia and indicate DR progression. Al-Nozha et al. [3] revealed that the overall DM prevalence was estimated to be $23.7 \%$ in Saudi Arabia. The prevalence of diabetes in 2013 was $8.3 \%$ and predicted to reach to $10.1 \%$ of the adult population by 2035 as reported by the World Health Organization [4]. A global review of DR reported that on an average approximately $34.6 \%$ of all diabetic patients has some DR form [5].

Oxidative damage is a critical factor involved in DR etiology. Reactive oxygen species (ROS) generated on account of hyperglycemic state through varied mechanisms tend to damage vital biomolecules like DNA, proteins, and lipid membranes damaging normal physiology of the cells [6]. Notably, the retina is highly vulnerable to damage by oxidative stress. Development of proliferative DR (PDR) and/or the diabetic macular edema can lead to visual impairment in DR patients [7]. Thus, the retinal blood vessel dysfunction is considered critical underlying the pathogenesis of DR.

The pathophysiology of DR progression is an intricate mechanism. Its initiation is related to hyperglycemia-induced ischemia and discharge of certain vasoactive chemicals, including vascular endothelial growth factor, that kindle the formation of new blood vessels from the retinal surface growing on the posterior wall of the vitreous chamber. As these newly formed blood vessels become fragile and have an immature nature, they can rupture. Consequently, blood and fluids leak out easily, resulting in vitreous hemorrhages. As the vitreous begins to constrict, it can result in retinal detachment, leading to vision loss [8]. DR characterized by angiogenesis that occurs during advanced stages is referred to as PDR. In contrast, another clinical subtype in the early stages is known as nonproliferative DR (NPDR), in which no new blood vessels are formed. Poor glycemic control and an alteration in antioxidant enzyme levels are strongly associated with DR development. Free radical attack accounts for oxidative damage in cells leading to loss of cell integrity and function. The overproduction of ROS and ROS accompanied with decreased antioxidant status (enzymes) results in enhanced cellular oxidation. Importantly, the cells are inclined for oxidative damage due to poor regulation by the antioxidant enzymes and this may be responsible for DM progression and its complications. The 2 prominent antioxidant enzymes involved are superoxide dismutase (SOD) and catalase (CAT). The disproportionation of the superoxide radical catalyzed by SOD results in generation of molecular oxygen $\left(\mathrm{O}_{2}\right)$ or hydrogen peroxide $\left(\mathrm{H}_{2} \mathrm{O}_{2}\right)[9,10]$. CAT is a common enzyme that catalyzes the decomposition of $\mathrm{H}_{2} \mathrm{O}_{2}$ to water and $\mathrm{O}_{2}$. The activity of CAT can reflect the ability of cells to remove ROS and the resistance to oxidative damage. It has been hypothesized that oxidative stress or poor antioxidant defense plays a crucial role in DR progression. Thus, this study aimed to evaluate the oxidative status (i.e., serum SOD and CAT activity) in patients with different stages of DR and evaluate the association between oxidative stress/antioxidant markers and DR severity in these subjects.

\section{Materials and Methods}

This cross-sectional study included 3 groups: subjects with T1DM $(n=17)$; T2DM $(n=96)$, and nondiabetic controls $(n=35)$ aged between 21 and 60 years. Subjects were recruited from the retinal clinics, Department of Ophthalmology, King Abdulaziz University Hospital (KAUH), Riyadh, Saudi Arabia. College Ethical Committee approved the study (CAMS 013-3839), and all participants included in the study signed the informed consent. Based on the exclusion criteria, samples were further screened to meet the required criteria and were subjected to further analysis. Subjects were divided into 2 subgroups based on the DR severity mild-to-severe NPDR and PDR.

Diabetic subjects with 10 -year history of diabetes with HbAlc $>6.5 \%$ and reduced insulin levels were categorized as type 1 and type 2 . Controls were healthy subjects with best-corrected visual acuity (VA) 20/40 and had no hypertension or hyperglycemia, no vascular disease, and no hyperlipidemia or any ocular diseases (e.g., hemorrhage and neovascularization). Diabetic subjects with glaucoma, liver disease, severe nephropathy, cancer, acute or chronic infections, fever, congestive heart failure, use of oral antioxidant supplements, or smoking history were excluded from the study.

\section{Ophthalmologic Examinations}

All subjects underwent ophthalmologic examinations including VA, intraocular pressure (IOP) measurement ( $\mathrm{mm} \mathrm{Hg}$ ), and fundus examination performed using slit lamp and ophthalmoscopy, as per the standard guidelines [11]. VA was determined using tumbling $\mathrm{E}$ chart and expressed in decimal notation as logarithm of the minimum angle of resolution (logMAR). The severity of DR was graded based on an abbreviated summary of the ETDRS 
Table 1. Baseline characteristics of the study groups

\begin{tabular}{|c|c|c|c|}
\hline & Control $(N=35)$ & $\mathrm{T} 1 \mathrm{DM}(N=17)$ & $\mathrm{T} 2 \mathrm{DM}(N=96)$ \\
\hline \multicolumn{4}{|l|}{ Age, $n(\%)$, years } \\
\hline$<20$ & $18.43 \pm 2.44(20)$ & $20 \pm 0.0(5.9)$ & $12 \pm 0.00(1)$ \\
\hline $21-40$ & $26 \pm 6.13(48.6)$ & $32 \pm 4.47(64.7)$ & $38 \pm 0.00(3.1)$ \\
\hline $41-60$ & $50.3 \pm 5.33(28.6)$ & $46 \pm 6.65(29.4)$ & $54.31 \pm 4.06(51)$ \\
\hline$<61$ & $64 \pm 0.00(2.9)$ & - & $67.7 \pm 6.3(44.8)$ \\
\hline Male sex, $n(\%)$ & $7(20)$ & $5(29.4)$ & $38(39.6)$ \\
\hline \multicolumn{4}{|c|}{ Duration of diabetes, $n(\%)$, years } \\
\hline$<10$ & - & $14(82.4)$ & $2(2.1)$ \\
\hline $10-25$ & - & $3(17.6)$ & $85(88.5)$ \\
\hline$\geq 26$ & - & & $9(9.4)$ \\
\hline \multicolumn{4}{|l|}{ Type of DR, $n(\%)$} \\
\hline Normal & - & - & $14(14.6)$ \\
\hline Mild NPDR & - & - & $6(6.3)$ \\
\hline Moderate NPDR & - & $1(5.9)$ & $29(30.2)$ \\
\hline Severe NPDR & - & $2(11.8)$ & $9(9.4)$ \\
\hline PDR & - & $14(82.4)$ & $38(39.6)$ \\
\hline \multicolumn{4}{|l|}{ VA (logMAR), $n(\%)$} \\
\hline $0.5-1.00$ & $34(97.1)$ & $3(17.6)$ & $29(30.2)$ \\
\hline$<0.5$ & $1(2.9)$ & $14(82.4)$ & $67(69.8)$ \\
\hline \multicolumn{4}{|l|}{ IOP, $n(\%), \mathrm{mm} \mathrm{Hg}$} \\
\hline $10-20$ & $35(100)$ & $12(70.6)$ & $73(76)$ \\
\hline$>20$ & & $5(29.4)$ & $23(24)$ \\
\hline
\end{tabular}

DR, diabetic retinopathy; NPDR, nonproliferative DR; PDR, proliferative DR; VA, visual acuity; IOP, intraocular pressure; T1DM, type 1 diabetes mellitus; T2DM, type 2 diabetes mellitus.

Table 2. Biochemical characteristics of the study group

\begin{tabular}{lcccc}
\hline & Control & T1DM & T2DM & $p$ value \\
\hline HbA1c, \% & $5.419 \pm 0.556^{\mathrm{a}}$ & $9.225 \pm 0.556^{\mathrm{a}}$ & $9.38 \pm 1.93^{\mathrm{a}}$ & 0.0002 \\
$\mathrm{TC}, \mathrm{mg} / \mathrm{dL}$ & $4.67 \pm 0.69$ & $4.5 \pm 0.36$ & $4.81 \pm 0.99$ & 0.31 \\
$\mathrm{HDL}-\mathrm{C}, \mathrm{mg} / \mathrm{dL}$ & $1.53 \pm 0.34 \mathrm{a}$ & $1.29 \pm 0.50$ & $1.17 \pm 0.29^{\mathrm{b}}$ & $<0.05$ \\
LDL-C, mg/dL & $2.41 \pm 0.89$ & $2.26 \pm 0.86$ & $2.44 \pm 1.16$ & 0.78 \\
TG, mg/dL & $0.89 \pm 0.41^{\mathrm{a}}$ & $1.1 \pm 0.43$ & $2.16 \pm 0.97^{\mathrm{a}}$ & 0.0002 \\
Antioxidant enzymes & & & & 0.31 \\
$\quad$ SOD (inhibition rate \%) & $57.26 \pm 0.16$ & $54.74 \pm 0.06$ & $49.51 \pm 0.17$ & 0.13 \\
\multicolumn{1}{c}{ CAT, U/mL } & $20.17 \pm 4.62$ & $16.14 \pm 6.05$ & $17.53 \pm 3.93$ & \\
\hline
\end{tabular}

T1DM, type 1 diabetes mellitus; T2DM, type 2 diabetes mellitus; SOD, superoxide dismutase; CAT, catalase; HbA1c, glycated hemoglobin. Values sharing the same superscript are significant at $p<0.0001$; different superscript ( $\mathrm{a}$ and $\mathrm{b}$ ) at $p<0.05$.

criteria; 0 = no retinopathy; $1=$ mild NPDR; $2=\operatorname{mNPDR} ; 3=$ severe NPDR; 4 = very severe NPDR; 5 = early PDR; 6 = high-risk PDR; and $7=$ PDR [12].

Analysis of Biochemical Markers and Antioxidant Enzymes

Blood samples were collected from subjects after $12 \mathrm{~h}$ of fasting. Overall, $8 \mathrm{~mL}$ blood was collected via venipuncture into labeled sterile vacutainers, and $1 \mathrm{~mL}$ was collected into an EDTA-coated tube for HbAlc analysis. All biochemical parameters, including HbA1c and lipid profile parameters were analyzed using a fully automatic analyzer (Roche Cobas C-702, Germany).

\section{Measurement of SOD Activity}

Activity of SOD was measured using the SOD Assay Kit-WST (19160; Sigma). SOD activity was determined following the manual instructions and reported as percentage inhibition rate. 


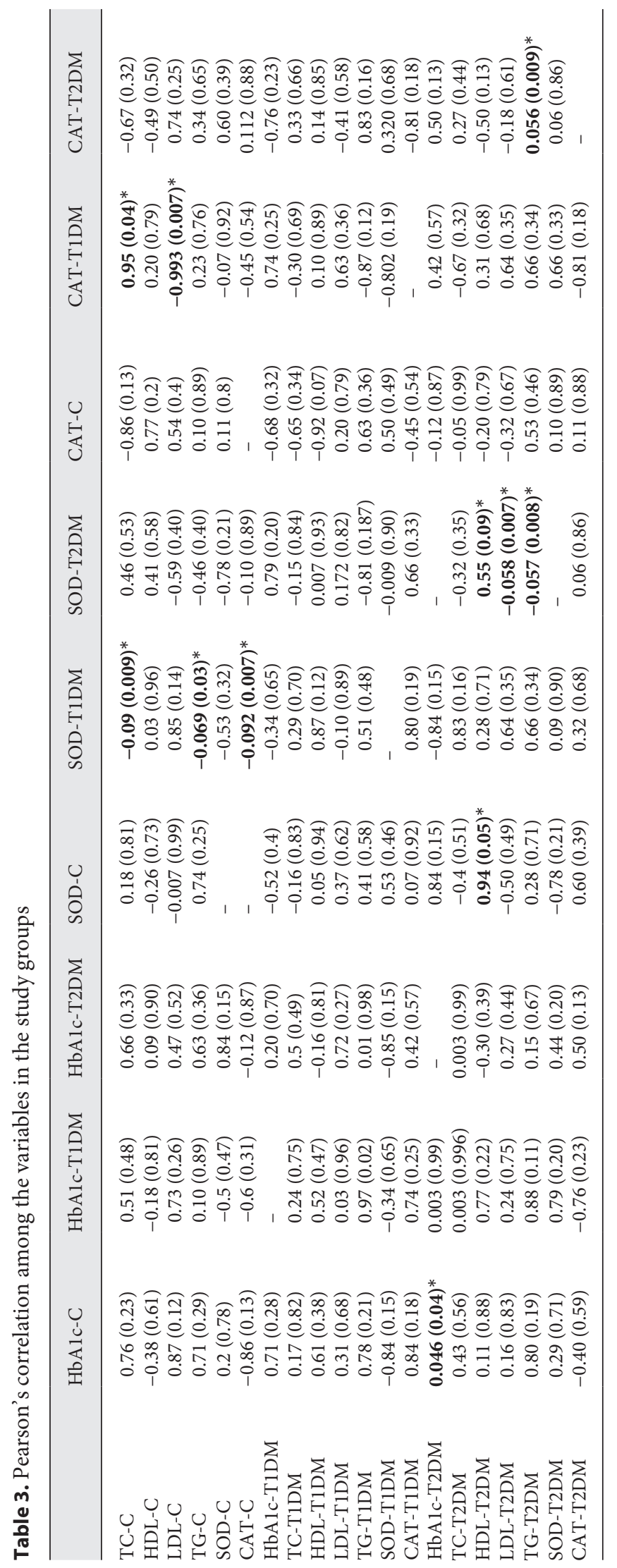

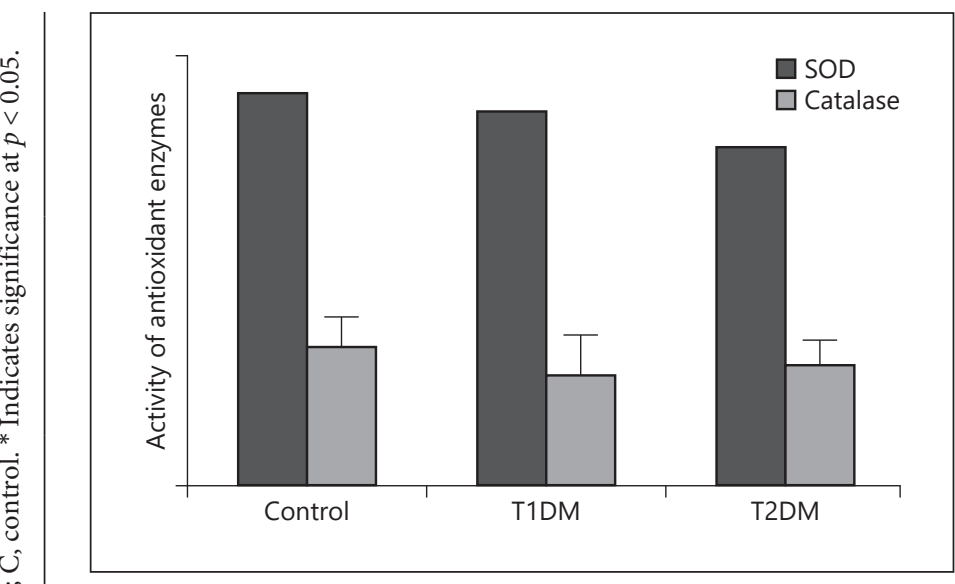

Fig. 1. Serum antioxidant enzyme levels in the 3 groups. SOD, superoxide dismutase; CAT, catalase; T1DM, type 1 diabetes mellitus; T2DM, type 2 diabetes mellitus.

Measurement of CAT Activity

Serum CAT activity was measured using a CAT colorimetric activity kit (EIACATC; Invitrogen), and CAT activity is expressed as $\mathrm{U} / \mathrm{mL}$.

\section{Statistical Analysis}

SPSS software version 22 was used for statistical analysis. Analysis of covariance was used to compare serum biochemical marker levels between controls and diabetic patients. The degree of retinopathy was coded and analyzed using 5 categorical variables $(1=$ no retinopathy, $2=$ mild NPDR, $3=$ moderate NPDR, $4=$ severe NPDR, $5=$ PDR. $p<0.05$ were considered statistically significant. The correlation between serum biochemical markers against retinopathy severity was analyzed using multiple linear regression. Significant differences between DR severity and antioxidant enzymes in NPDR and PDR subgroups were analyzed using the Kruskal-Wallis test followed by Dunn's test.

\section{Results}

The preliminary characteristics of the study population are represented in Table 1 . The mean ages \pm standard deviation of the T1DM, T2DM, and control groups were $32 \pm 4.47,54.31 \pm 4.06$, and $26 \pm 6.13$ years, respectively. Ocular examination showed that 99 subjects were positive DR (+) (T1DM-17; T2DM-82) and 49 subjects were negative DR (-) (control-35; T2DM-14). IOP was in upper physiological limits in controls and most diabetic patients in both groups. Around $82.4 \%$ of T1DM and $88.5 \%$ T2DM patients had diabetes for 10-20 years and exhibited severe PDR in T1DM and T2DM (82.4 and 39.6\%, respectively). 
Fig. 2. Correlation between DR severity and antioxidant enzymes. DR, diabetic retinopathy; SOD, superoxide dismutase; CAT, catalase.

Fig. 3. Correlation between $\mathrm{HbA} 1 \mathrm{c}$ and $\mathrm{DR}$ severity. DR, diabetic retinopathy; HbAlc, glycated hemoglobin.
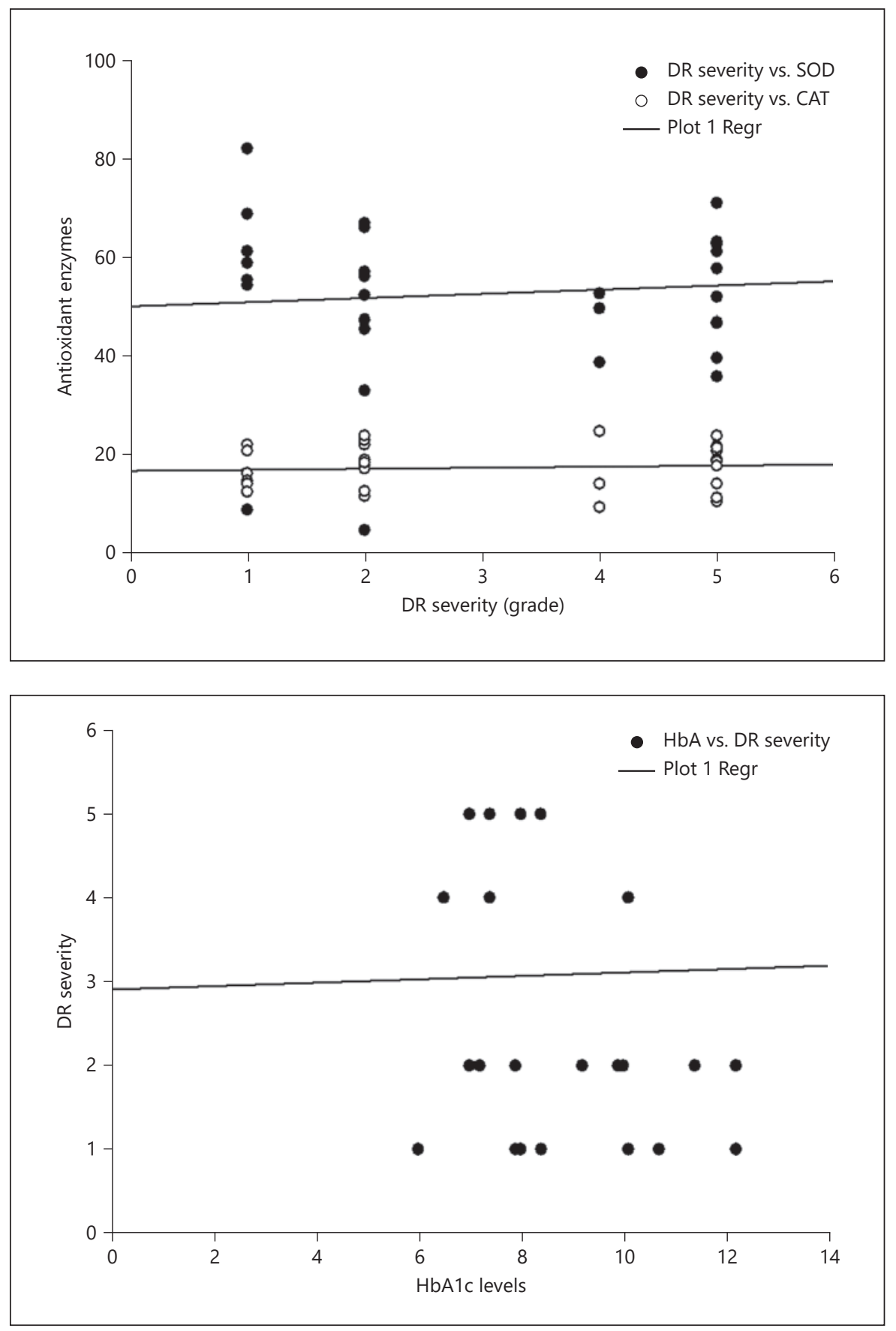

Serum biochemical marker levels and antioxidant status are shown in Table 2. Diabetic subjects had higher HbA1c, TG, and LDL-C levels than the control group with statistically significant difference in levels of $\mathrm{HbAlc}$ and TG at $p<0.001$. In contrast, HDL-C levels were lower in diabetic patients than in controls $(p<0.05)$. The antioxidant activity of SOD decreased in T1DM (54.74 \pm $0.06)$ and $\mathrm{T} 2 \mathrm{DM}(49.51 \pm 0.17)$ compared to controls
$(57.26 \pm 0.16)$. Also, the diabetic groups exhibited diminished activity of CAT enzyme. However, these differences in activity and level were not significant. Pearson's correlation among the variables in the study groups revealed HDL positively correlated with SOD in diabetic subjects. Furthermore, LDL was found to be negatively correlated with TG and LDL-C among diabetic subjects at $p<0.05$ (Table 3). The comparison of the SOD and CAT levels in 
Table 4. Status of serum antioxidant enzymes in diabetic patients with/without retinopathic changes

\begin{tabular}{llr}
\hline Antioxidant enzyme & \multicolumn{2}{l}{ Diabetic subjects } \\
\cline { 2 - 3 } & DR $(-)$ & DR $(+)$ \\
\hline SOD (inhibition rate \%) & $49.47 \pm 14.4^{\mathrm{a}}$ & $48.94 \pm 18.8^{\mathrm{a}}$ \\
CAT, U/mL & $16.25 \pm 3.8^{\mathrm{a}}$ & $17.7 \pm 4.80^{\mathrm{a}}$ \\
\hline
\end{tabular}

DR, diabetic retinopathy; SOD, superoxide dismutase; CAT, catalase. ${ }^{a}$ Values sharing the same superscript are significant at $p<0.001$.

the studied groups is illustrated in Figure 1. The correlations between antioxidant levels and severity of DR in the diabetic group were investigated and showed no significant correlation between antioxidant level (SOD and CAT) and severity of DR (Fig. 2). The correlation between $\mathrm{HbAlc}$ and severity of DR is depicted in Figure 3. Table 4 shows the differences in the antioxidant enzymes among the diabetic group with or without retinopathic changes. SOD and CAT levels were observed to decrease significantly at $p<0.001$. Increased levels of HbA1c were significantly associated with decreased SOD in both the groups. However, CAT levels varied insignificantly to $\mathrm{HbA1c}$ in diabetic patients with and without retinopathic changes.

Diabetic groups were subdivided into NPDR and PDR based on the grades of DR severity. Serum levels of HbA1c, SOD, and CAT in the subgroups are depicted in Figure 4. Regression graph showing the correlation between severity of DR and antioxidant enzymes in the NPDR and PDR subgroups is represented in Figure 5. Among the NPDR and PDR subgroups, the activities of the 2 antioxidant enzymes varied significantly with DR severity $(p<0.05$; $Q=5.5$ and 3.9, and 5.73 and 3.69, respectively). HbAlc levels varied significantly with superoxide dismutase in the NPDR and PDR subgroups $(p<0.05)$. Furthermore, the Kruskal-Wallis test performed to analyze NPDR and PDR subgroups yielded informative results. Both antioxidant enzyme levels were significantly different $(p<0.05)$ between the groups defined by the severity of DR. Serum levels of HbA1C, SOD, and CAT were analyzed by the Shapiro-Wilk normality test and Kruskal-Wallis test followed by multiple comparison using Dunn's test.

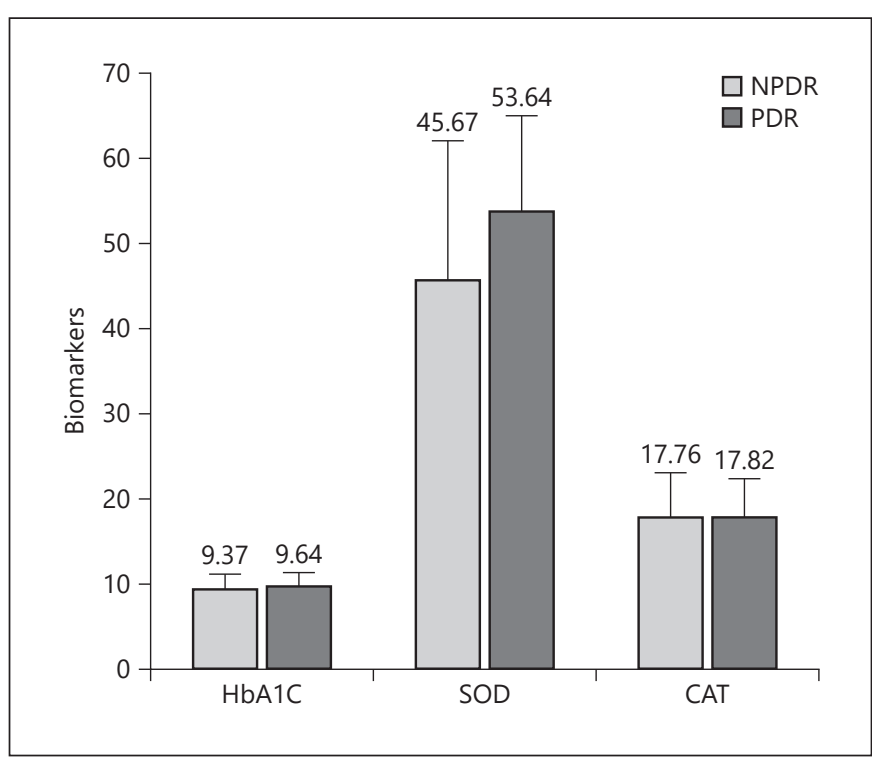

Fig. 4. Status of biochemical markers in DR subgroups - NPDR and PDR. DR, diabetic retinopathy; NPDR, nonproliferative DR; PDR, proliferative DR; HbA1c, glycated hemoglobin; SOD, superoxide dismutase; CAT, catalase; HbAlc, glycated hemoglobin.

\section{Discussion}

Oxidative stress can contribute to the pathogenesis of DR. Interestingly, we observed reduced antioxidant activity in DR patients compared to healthy nondiabetic subjects. Increased $\mathrm{HbA1c}, \mathrm{TG}$, and LDL-C levels were found in subjects with both DM types compared to nondiabetic control. Hyperglycemia-induced oxidative stress is considered an important factor that contributes to DR. Schematic diagram of role of oxidative stress in retinopathy is depicted in Figure 6.

Oxidative damage, as introduced earlier, is the result of free radical attack that leads to a loss of structure and function of cells and their components [13]. Numerous mechanisms are considered to play a crucial role in etiology underlying DR viz synthesis of advanced glycation end products, linked with the overproduction of ROS [14]. Increased free radical or ROS levels because of an imbalance in the oxidation-reduction cycles in the biological system eventually lead to the destruction of the antioxidant system composed of prominent antioxidant enzymes. Antioxidant enzyme levels critically affect the susceptibility of cells to oxidative stress and may also be linked to the progress of diabetes-related complications including retinopathy. Additionally, the hyperglycemic state can exacerbate the effects of oxidative damage and 
Fig. 5. Regression graph showing the correlation between DR severity and antioxidant enzymes in the NPDR and PDR subgroups. DR, diabetic retinopathy; NPDR, nonproliferative DR; PDR, proliferative DR; SOD, superoxide dismutase; CAT, catalase.

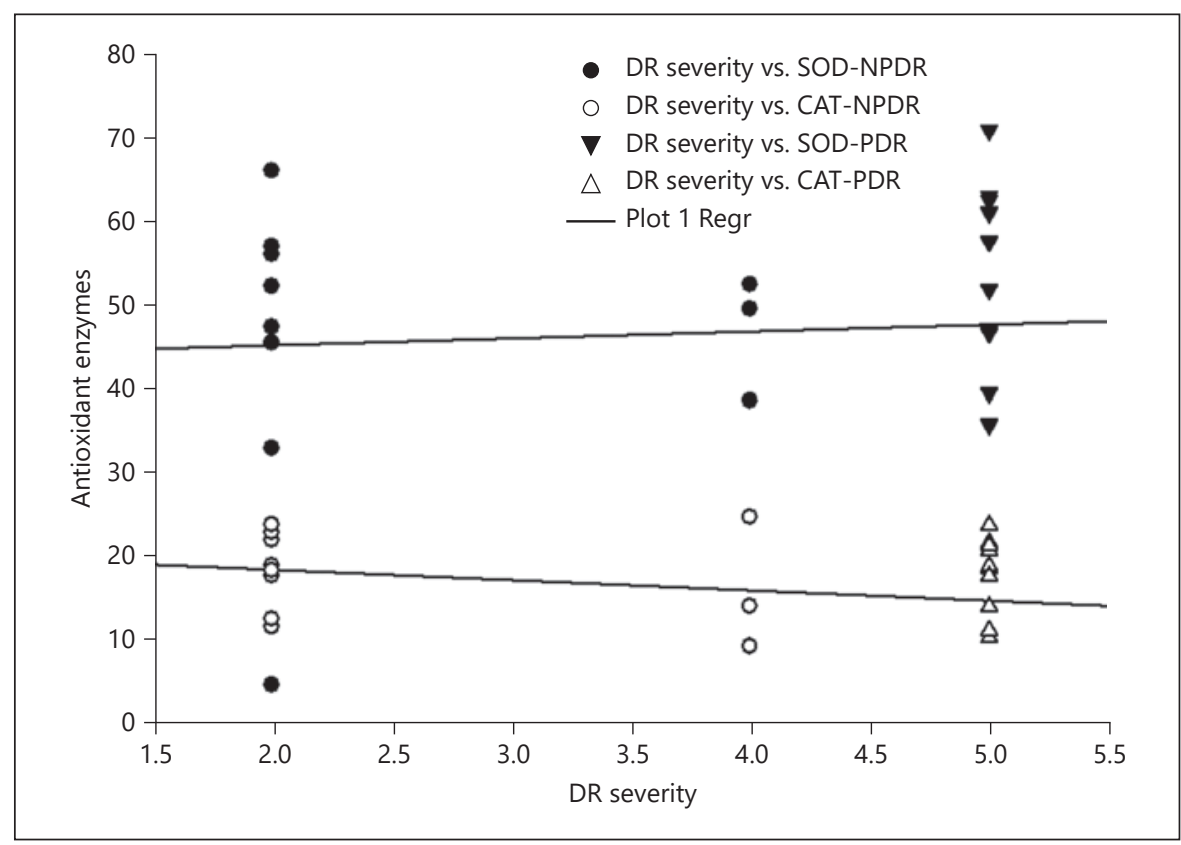

lead to DR. SOD is the major scavenger of ROS, which are associated with diabetic complications. It is catalytically involved in the production of the $\mathrm{H}_{2} \mathrm{O}_{2}$ and $\mathrm{O}_{2}$ from superoxide anions. CAT regulates $\mathrm{H}_{2} \mathrm{O}_{2}$ metabolism, and diminished CAT levels can have deleterious effects on cellular components. Under CAT-deficient conditions, enhanced oxidation in pancreatic beta cells could lead to beta-cell dysfunction and eventually to diabetes [15]. Parallel to present study, Pan et al. [16] studied oxidation process in DR and found same correlation. The observations in the current study, hyperglycemia with poor antioxidant enzymes in T2 diabetic patients are similar to as reported earlier [17, 18]. Similarly, Alghazeer et al. [19] reported decreased SOD levels and CAT activity in T1DM patients compared to nondiabetic controls. It is speculated that the increase in enzymatic glycation due to glucose overload may account for diminished CAT activity in diabetic patients. DR progression to PDR was correlated with increased oxidative damage and decreased antioxidant activity in previous reports [20]. The nonsignificant decrease in SOD levels in the diabetic group is in accordance to the results reported by Gurler et al. [21].

Pearson's correlation was used to study the correlation between antioxidant status and severity of DR in diabetes patients. There was no correlation between antioxidant markers and severity of DR in either type of diabetic patients. Exploring the role of antioxidant enzymes, the diabetic groups were divided into 2 subgroups, NPDR and

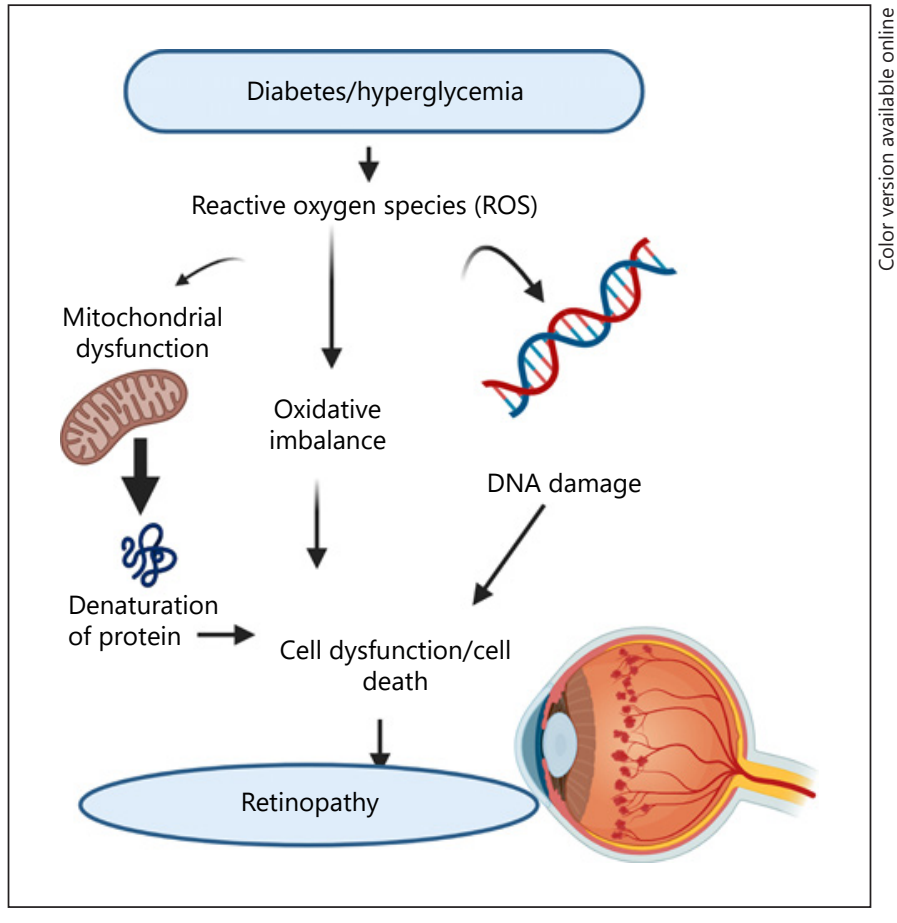

Fig. 6. Schematic diagram of role of oxidative stress in retinopathy. ROS, reactive oxygen species. 
PDR, based on the severity of DR. The comparison of $\mathrm{HbA1c}, \mathrm{SOD}$, and CAT levels between the 2 subgroups NPDR and PDR is depicted in Figure 4. The new insight was the levels of HbAlc varied significantly $(p<0.05)$ with SOD in NPDR and PDR, whereas levels of HbA1C and CAT were statistically significant only in the PDR subgroup. These findings are in agreement with earlier reports [22]. Certainly, activities of these 2 antioxidant enzymes - SOD and CAT - were found to be involved in $\mathrm{DR}$ progression. Increased $\mathrm{HbAlc}$ thus reflected the cause of oxidative damage by altering SOD and CAT distinctly and mediating the subsequent progression of NPDR to PDR. Thus, the role of antioxidant enzymes in DR progression was conspicuous in our study. The results obtained in the present study revealed the relative role of these enzymes in the onset of diabetes and to subsequent stages of DR. It is noteworthy that serum SOD and CAT activity might be a biomarker for DR screening and evaluation of the clinical severity of DR in T2 diabetic patients. However, further elaborative investigations are needed to explicitly understand such associations.

The main study finding was increased $\mathrm{HbAlc}$ levels, poor antioxidant status along with diminished VA in diabetic patients. Approximately $82.4 \%$ of $\mathrm{T} 1$ diabetic patients and $69.8 \%$ of T2 diabetic patients had VA $<0.5$ logMAR compared to nondiabetic controls with $97.1 \%$ of subjects in the range of $0.5-1.00 \log$ MAR. It is noteworthy that the IOP for all nondiabetic controls was within the normal range $(10-20 \mathrm{~mm} \mathrm{Hg})$, while $30 \%$ of $\mathrm{T} 1$ and $24 \%$ of T2 diabetic patients had an IOP $>20 \mathrm{~mm} \mathrm{Hg}$. The diabetic group exhibited higher IOP than controls in the upper physiological limit. Presumably, elevated IOP could lead to the death of ganglion cells in the retina which has been documented in previous reports $[23,24]$. Furthermore, raised IOP levels can lead to the inception of optic neuropathy, which gradually destroys the optic nerve head by mechanical compression. Ultimately, progressive loss of optic nerve fibers manifested by vision loss can be caused by such compression. In conclusion, the present findings support the hypothesis that oxidative stress and decreased antioxidant defenses are associated with DR progression to PDR.

The major findings and strength of the current study were the role of the antioxidant enzymes in progression of DR; NPDR to PDR was evident. HbA1c as a predisposing factor in progression of DR following generation of imbalance of the oxidant-antioxidant system reflected by altered SOD and CAT added to a better understanding of the etiopathological mechanism underlying DR.
However, the study has some limitations. First, although the association of oxidative stress in diabetic progression to DR is confirmed, the evaluation of oxidative stress markers as a comparative study from other ocular diseases would have added additional information to the outcome of the present investigation. Second, study reports association on a small sample size, and hence, larger studies investigating a large number samples are needed.

\section{Acknowledgement}

The authors are thankful to the Research Support and Services Unit, King Saud University, for technical support.

\section{Statement of Ethics}

The study was approved by the Ethics Committee (Approval No. CAMS 013-3839), King Saud University. All participants included in the study signed the informed consent.

\section{Conflict of Interest Statement}

All authors declare no conflict of interest.

\section{Funding Sources}

The authors extend their appreciation to the Deanship of Scientific Research at King Saud University for finding this work through the Undergraduate Student's Research Support Program, project number: URSP-3-18-72.

\section{Author Contributions}

Conceived and designed the experiments: Kholoud Bokhary and Manal Abudawood; performed the experiments: Afnan Bakhsh, Shatha I. Alhammad, and Rawan Aleyadhi; literature search: Hajera Tabassum and Feda Aljaser; data analysis: Kholoud Bokhary; statistical analysis: Hajera Tabassum; manuscript preparation: Kholoud Bokhary and Hajera Tabassum; manuscript editing and manuscript review: Feda Aljaser, Faisal Almajed, and Roua Alsubki. 


\section{References}

1 Koenig RJ, Peterson CM, Jones RL, Saudek C, Lehrman M, Cerami A. Correlation of glucose regulation and hemoglobin AIc in diabetes mellitus. N Engl J Med. 1976;295(8):417-20.

2 Armstrong D, Ueda T, Ueda T, Aljada A, Browne R, Fukuda S, et al. Lipid hydroperoxide stimulates retinal neovascularization in rabbit retina through expression of tumor necrosis factor-alpha, vascular endothelial growth factor and platelet-derived growth factor. Angiogenesis. 1998;2(1):93-104.

3 Al-Nozha MM, Al-Maatouq MA, Al-Mazrou YY, Al-Harthi SS, Arafah MR, Khalil MZ, et al. Diabetes mellitus in Saudi Arabia. Saudi Med J. 2004;25(11):1603-10.

4 World Health Organization (WHO). Diabetes, fact sheet WHO/312; 2013. Available from: http://www.who.int/mediacentre/factsheets/fs312/en/ Accessed 2020 Jan.

5 Yau JW, Rogers SL, Kawasaki R, Lamoureux EL, Kowalski JW, Bek T, et al. Global prevalence and major risk factors of diabetic retinopathy. Diabetes Care. 2002;35(3):556-64.

6 Oguntibeju OO. Type 2 diabetes mellitus, oxidative stress and inflammation: examining the links. Int J Physiol Pathophysiol Pharmacol. 2019;11(3):45-63.

7 Cohen SR, Gardner TW. Diabetic retinopathy and diabetic macular edema. Dev Ophthalmol. 2015;55:137-46.

8 Safi SZ, Qvist R, Kumar S, Batumalaie K, Ismail IS. Molecular mechanisms of diabetic retinopathy, general preventive strategies, and novel therapeutic targets. Biomed Res Int. 2014;2014:801269.
9 Brand MD, Buckingham JA, Esteves TC, Green K, Lambert AJ, Miwa S, et al. Mitochondrial superoxide and aging: uncouplingprotein activity and superoxide production. Biochem Soc Symp. 2004;7(71):203.

10 Wang L, Gong H, Lv R, Wu Y, Wang T. A one-pot aqueous synthesis of high-luminescent thiol-capped CdTe and its bioapplication. J Nanosci Nanotechnol. 2010;10(8): 5106-10.

11 American Academy of Ophthalmology Preferred Practice Patterns Committee. Preferred practice pattern guidelines. Comprehensive adult medical eye evaluations. San Francisco: American Academy of Ophthalmology; 2010.

12 Early Treatment Diabetic Retinopathy Study Research Group. Photocoagulation for Diabetic macular edema: ETDRS report number 1. Arch Ophthalmol. 1985;103:1796-806.

13 Calderon GD, Juarez OH, Hernandez GE Punzo SM, De la Cruz ZD. Oxidative stress and diabetic retinopathy: development and treatment. Eye. 2017;31(8):1122-30.

14 Brownlee M. The pathobiology of diabetic complications: a unifying mechanism. Diabetes. 2005;54(6):1615-25.

15 Ullah A, Khan A, Khan I. Diabetes mellitus and oxidative stress: a concise review. Saudi Pharm J. 2016;24:547-53.

16 Pan HZ, Zhang H, Chang D, Li H, Sui H. The change of oxidative stress products in diabetes mellitus and diabetic retinopathy. $\mathrm{Br} \mathrm{J}$ Ophthalmol. 2008;92(4):548-51.

17 Ngaski AA. Correlation of antioxidants enzymes activity with fasting blood glucose in diabetic patients in Sokoto, Nigeria. J Adv Med Res. 2018;25:1-6.
18 Palekar AV, Ray KS. Oxidative stress in patients with diabetes mellitus. J Diabetes Metab Disord Control. 2016;3:138-43.

19 Alghazeer R, Alghazir N, Awayn N, Ahtiwesh $\mathrm{O}$, Elgahmasi S. Biomarkers of oxidative stress and antioxidant defense in patients with type 1 diabetes mellitus. Ibnosina J Med Biomed Sci. 2018;10:198-204.

20 Mancino R, Di Pierro D, Varesi C, Cerulli A, Feraco A, Cedrone C, et al. Lipid peroxidation and total antioxidant capacity in vitreous, aqueous humor, and blood samples from patients with diabetic retinopathy. Mol Vis. 2011;17:1298-304.

21 Gurler B, Vural H, Yilmaz N, Oguz H, Satici A, Aksoy N. The role of oxidative stress in diabetic retinopathy. Eye. 2000;5:730-5.

22 Beyazyıldız E, Cankaya AB, Ergan E, Anayol MA, Ozdamar Y, Sezer S, et al. Changes of total antioxidant capacity and total oxidant status of aqueous humor in diabetes patients and correlations with diabetic retinopathy. Int J Ophthalmol. 2013;6(4):531-6.

23 Guo L, Moss SE, Alexander RA, Ali RR, Fitzke FW, Cordeiro MF. Retinal ganglion cell apoptosis in glaucoma is related to intraocular pressure and IOP-induced effects on extracellular matrix. Invest Ophthalmol Vis Sci. 2005; 46(1):175-82.

24 Soto I, Howell GR, John CW, Kief JL, Libby RT, John SW. DBA/2J mice are susceptible to diabetic nephropathy and diabetic exacerbation of IOP elevation. PLoS One. 2014;9(9): e107291. 